Pesq. Vet. Bras. 30(8):631-636, agosto 2010

\title{
Polioencefalomalacia experimental induzida por amprólio em bovinos ${ }^{1}$
}

\author{
Ana Paula A. Nogueira² ${ }^{2}$ Roosevelt I.C. Souza ${ }^{2}$, Bethânia S. Santos ${ }^{2}$, Ana Paula \\ Pinto $^{2}$, Nickolly L.K.S. Ribas ${ }^{3}$, Ricardo A.A. Lemos ${ }^{3}$ e Fabiano J.F. de Sant'Ana ${ }^{4 *}$
}

\begin{abstract}
Nogueira A.P.A., Souza R.I.C., Santos B.S., Pinto A.P., Ribas N.L.K.S., Lemos R.A.A. \& Sant'Ana F.J.F. 2010. [Experimentally amprolium-induced polioencephalomalacia in cattle.] Polioencefalomalacia experimental induzida por amprólio em bovinos. Pesquisa Veterinária Brasileira 30(8):631-636. Faculdade de Medicina Veterinária e Zootecnia, Universidade Federal de Mato Grosso do Sul, Campo Grande, MS 79070-900, Brazil. E-mail: santanafjf@yahoo.com

In order to establish an experimental model for the study of the etiology, pathology, and pathogenesis of polioencephalomalacia (PEM) in cattle, the condition was induced into four steers by oral administration of amprolium at daily doses of 500 and $350 \mathrm{mg}$ per $\mathrm{kg}$ of body weight respectively for 22 and 26-28 days. All steers died spontaneously or were euthanized in extremis after being sick for 4-7 days. Three steers that received the drug at $1,000 \mathrm{mg} / \mathrm{kg}$ and two that received $500 \mathrm{mg} / \mathrm{kg}$ died spontaneously with acute or subacute clinical signs and without lesions and signs of PEM. In those steers in which PEM was reproduced, the neurological signs included marked apathy, incoordination, sawhorse stance, occasional falls, hyperexcitability, muscle tremors, blindness, grinding of teeth, strabismus, nystagmus, mydriasis, opisthotonus, and lateral recumbency with paddling movements. Main gross lesions were restricted to the brain and included swelling, flattening, softening and yellow discoloration of the cerebral circumvolutions. Histologically, there was segmental laminar neuronal necrosis (red neurons) associated with edema, swelling of endothelial cells, cleavage of laminar neuronal layers or between gray and white matter and moderate to severe infiltration by foamy macrophages (gittercells). These changes were more marked in the frontal, parietal and occipital telencephalic lobes. Additionally, similar and moderate lesions were detected in the midbrain and hippocampus. Neuronal necrosis and edema affected uniformly the neurons layers of the grey matter of the frontal, parietal and occipital lobes. This experimental model of PEM with oral administration of amprolium may be useful for the study in cattle, as previously observed in sheep.
\end{abstract}

INDEX TERMS: Diseases of cattle, neuropathology, amprolium, polioencephalomalacia, experimental model.

${ }^{1}$ Recebido em 22 de março de 2010.

Aceito para publicação em 30 de março de 2010

Parte da Dissertação de Mestrado da primeira autora. Projeto financiado pela Fundação de Apoio ao Desenvolvimento do Ensino, Ciência e Tecnologia do Estado de Mato Grosso do Sul (Fundect), Edital Universal 07/2007.

2 Programa de Pós-Graduação em Ciência Animal, Faculdade de Medicina Veterinária e Zootecnia (Famez), Universidade Federal de Mato Grosso do Sul (UFMS), Av. Filinto Müller s/n, Cidade Universitária, Campo Grande, MS 79070-900, Brasil.

${ }^{3}$ Departamento de Medicina Veterinária, (Famez), Universidade Federal de Mato Grosso do Sul(UFMS), Campo Grande, MS.

${ }^{4}$ Laboratório de Patologia Veterinária, Universidade Federal de Goiás (UFG), Campus Jataí, BR 364 Km 192, Jataí, GO 75800-000, Brasil.

*Autor para correspondência: santanafjf@yahoo.com
RESUMO.- Para estabelecer um modelo experimental para o estudo da etiologia, patologia e patogênese da polioencefalomalacia (PEM) em bovinos, a condição foi induzida em quatro novilhos pela administração oral de amprólio nas doses diárias de 500 e $350 \mathrm{mg} / \mathrm{kg}$ de peso vivo, respectivamente por 22 e 26 28 dias. Todos os bovinos morreram espontaneamente ou foram eutanasiados in extremis após um curso clínico de 4-7 dias. Três bovinos que receberam $1.000 \mathrm{mg} / \mathrm{kg}$ de amprólio e dois que receberam $500 \mathrm{mg} / \mathrm{kg}$ morreram espontaneamente com quadro clínico agudo a subagudo sem desenvolverem sinais e lesões de PEM. Nos novilhos que PEM foi reproduzida, os sinais neurológicos incluíram marcada apatia, incoordenação, posição de cavalete, quedas ocasionais, hiperexcitabilidade, 
tremores musculares, cegueira, bruxismo, estrabismo, nistagmo, midríase, opistótono, decúbito lateral e movimentos de pedalagem. Os principais achados de necropsia eram restritos ao encéfalo e consistiam de tumefação, achatamento, amolecimento e amarelamento das circunvoluções cerebrais. Histologicamente, havia necrose neuronal segmentar e laminar (neurônios vermelhos) associada a edema, tumefação endotelial, separação das lâminas de neurônios do córtex telencefálico ou entre as substâncias cinzenta e branca e infiltração moderada a acentuada de macrófagos espumosos. Essas alterações eram mais acentuadas nos lobos telencefálicos frontal, parietal e occipital. Adicionalmente, lesões similares e moderadas foram detectadas no mesencéfalo e hipocampo. A necrose neuronal e o edema afetaram uniformemente as camadas de neurônios da substância cinzenta dos lobos telencefálicos frontal, parietal e occipital. Esse modelo experimental de PEM com administração oral de amprólio parece ser útil para o estudo da doença em bovinos, conforme observado anteriormente em ovinos.

TERMOS DE INDEXAÇÃO: Doenças de bovinos, neuropatologia, amprólio, polioencefalomalacia, modelo experimental.

\section{INTRODUÇÃO}

Polioencefalomalacia (PEM) é um diagnóstico morfológico para necrose com amolecimento (malacia) da substancia cinzenta (polio) do encéfalo. A etiologia e patogênese da PEM em ruminantes ainda é controversa. A deficiência de tiamina foi inicialmente considerada como causa de PEM em ruminantes (Jensen et al. 1956), mas várias outras causas têm sido incriminadas na PEM em ruminantes (Sant'Ana et al. 2009b) e em algumas dessas situações, a participação da tiamina na patogênese da PEM pode ser questionada, uma vez que não há alterações nas concentrações sanguíneas dessa substância.

Para o estudo da patogênese da PEM dos ruminantes, é indicado o estabelecimento de um modelo experimental do qual se conheçam detalhadamente a dose do agente causador, a evolução dos sinais clínicos, o tipo e a distribuição das alterações no encéfalo (Sant'Ana et al. 2009c). Embora existam trabalhos que mencionam alguns aspectos neuropatológicos da intoxicação por amprólio em ruminantes (Loew \& Dunlop, 1972, Markson et al. 1974, Morgan etal. 1975, Kasahara et al. 1989), pouco se conhece a respeito da caracterização morfológica das alterações produzidas por esse antagonista da tiamina, bem como sobre a distribuição dessas lesões no encéfalo. Como parte de estabelecer um modelo para o estudo da PEM em ruminantes, a reprodução da doença pela administração de amprólio foi realizada em ovinos (Sant'Ana et al. 2009c). O presente trabalho faz parte do seguimento deste estudo e objetiva descrever os achados clinicopatológicos e a distribuição das alterações no encéfalo em bovinos intoxicados experimentalmente com amprólio.

\section{MATERIAL E MÉTODOS}

Foram utilizados onze bovinos machos, de doze meses de idade, com pesos entre 110-293 kg, que foram everminados e numerados de 1-11 antes do início do experimento (Quadro 1). Durante o período de adaptação de 15 dias e por todo o experimento os bovinos foram mantidos em curral, onde recebiam feno de alfafa, água a vontade e $1 \%$ de peso vivo em concentrado. Os bovinos foram pesados no início do experimento. Diariamente era realizado exame físico visando principalmente a detecção de sinais clínicos neurológicos.

Os bovinos foram distribuídos aleatoriamente em três grupos experimentais (com três animais cada) e receberam amprólio (Amprolbase [40\% de amprólio] ${ }^{5}$ nas dosagens de 1.000 mg/kg/dia (Grupo 1, Bovinos 1-3), 500 mg/kg/dia (Grupo 2, Bovinos 4-6) e 350mg/kg/dia (Grupo 3, Bovinos 7-9) até a manifestação dos primeiros sinais clínicos neurológicos. Os Bovinos 10 e 11 não receberam amprólio, mas foram mantidos por todo o experimento nas mesmas condições que os outros nove novilhos, e serviram de controle. O amprólio era pesado diariamente em balança de precisão e acondicionado em sacos plásticos individuais que eram identificados com o número do bovino que receberia aquela porção. A administração do produto era feita via sonda esofágica. O amprólio era diluído em quantidade suficiente de água para viabilizar a passagem do produto pela sonda. Os bovinos experimentais morreram naturalmente ou foram submetidos à eutanásia in extremis. Todos os bovinos foram necropsiados, com exceção dos Bovinos 10 e 11 que foram mantidos vivos.

O encéfalo juntamente com o complexo gânglio de Gasser, rete mirabile carotídea e hipófise $(\mathrm{GRH})$, e fragmentos de diversos outros órgãos eram coletados e fixados em formol a $10 \%$. Após a fixação, eram realizados cortes transversais no encéfalo com intervalos de $1 \mathrm{~cm}$ para avaliação macroscópica. As seguintes seções eram preparadas para avaliação microscópica: cerebelo, bulbo na altura do óbex, ponte com pedúnculos cerebelares, mesencéfalo na altura dos colículos rostrais, tálamo, núcleos basais e hipocampo; foram também examinados os lados direito e esquerdo dos telencéfalos frontal, parietal e occipital. Adicionalmente, o exame histológico era realizado em um monobloco do complexo GRH. Todos os fragmentos eram processados rotineiramente para histologia, incluídos em parafina e corados pela hematoxilina-eosina (HE).

As alterações observadas no encéfalo foram graduadas de acordo com a intensidade numa escala de 0 (ausência de lesão) a 3 (lesão acentuada). Cada lesão tinha seu grau aferido de acordo com a média de percepção subjetiva de três patologistas que examinaram todos os casos. Foram avaliados o grau de intensidade e a distribuição nos diferentes locais examinados procurando-se pelas seguintes alterações histológicas: gliose focal, gliose difusa, tumefação de núcleos de células endoteliais, separação entre as lâminas de neurônios corticais ou entre as substâncias cinzenta e branca, edema do neurópilo, congestão, hemorragia, necrose neuronal (caracterizada por picnose nuclear e eosinofilia e encarquilhamento citoplasmáticos), neuronofagia, malacia (o termo malacia foi empregado aqui significando necrose de todas as células neuroectodérmicas com manutenção das células mesenquimais (i.é, células gitter, células endoteliais) que sobrevivem e participam do processo [Innes \& Saunders 1962]), lesão residual (estrutura cavitária formada por pequenos vasos e numerosas células gitter remanescentes) e infiltrado inflamatório perivascular e/ou meníngeo. A distribuição do edema e da necrose neuronal (neurônio vermelho) nas lâminas da substância cinzenta do telencéfalo era também determinada.

\footnotetext{
${ }^{5}$ Amprolbase ${ }^{\circledR}$, Laboratório Farmabase Saúde Animal, Av. Emílio Marconato 1000, Galpão A3, Chácara Primavera, Jaguariúna, SP 13820-000, www.farmabase.com.br
} 


\section{RESULTADOS}

Dos nove bovinos que receberam amprólio, seis morreram espontaneamente (Bovinos 1-5 e 7) e três foram eutanasiados in extremis (Bovinos 6, 8 e 9). Três quadros clínicos distintos foram observados, e na maioria dos casos, foram de acordo com a dosagem de amprólio administrada; o primeiro quadro, agudo a subagudo, foi observado nos bovinos do Grupo 1 e foi caracterizado por surgimento de sinais clínicos com poucos minutos a 6 dias após a administração do produto. A evolução clínica nesse grupo variou de 1 hora a 3 dias. Os sinais clínicos compreendiam dificuldade de locomoção, prostração e decúbito. O Bovino 3 apresentou melena e hemorragias multifocais cutâneas na barbela e no dorso.

No Grupo 2, dois bovinos adoeceram após 13-14 dias de administração de amprólio. Um desses bovinos (Bovino 5) morreu sem manifestar sinais clínicos e o outro (Bovino 4) teve curso clínico de dois dias caracterizado exclusivamente por epistaxe. $\mathrm{O}$ terceiro bovino desse grupo apresentou evolução clínica de 4 dias após 22 dias de administração do produto. Os sinais eram principalmente neurológicos e consistiram de incoordenação, quedas frequentes, cegueira, estrabismo, nistagmo, midríase, opistótono, decúbito lateral e movimento de pedalagem.

Os primeiros sinais clínicos observados nos bovinos do Grupo 3 surgiram 26-28 dias após a administração do amprólio. A evolução clínica variou de 5-7 dias. Os sinais eram especialmente neurológicos e incluíam apatia marcada (Fig.1), anorexia, posição de cavalete, hiperexcitabilidade, bruxismo, cegueira, nistagmo, tremores musculares, decúbito esternal e posteriormente lateral, opistótono e movimentos de pedalagem (Fig.2).

Os Bovinos 10 e 11 não apresentaram alterações clínicas.

Macroscopicamente, os bovinos do Grupo 1 e o Bovino 4 (Grupo 2) apresentaram alterações circulatórias, principalmente hemorragias em diversos órgãos do trato digestivo (Bovinos 1, 3 e 4), na pele (Bovino 3), traquéia (Bovino 2), subcutâneo, no endocárdio e na bexiga (Bovino 3) e hemoperitônio (Bovino 4) (Quadro 1). Congestão e edema pulmonar foram obser-

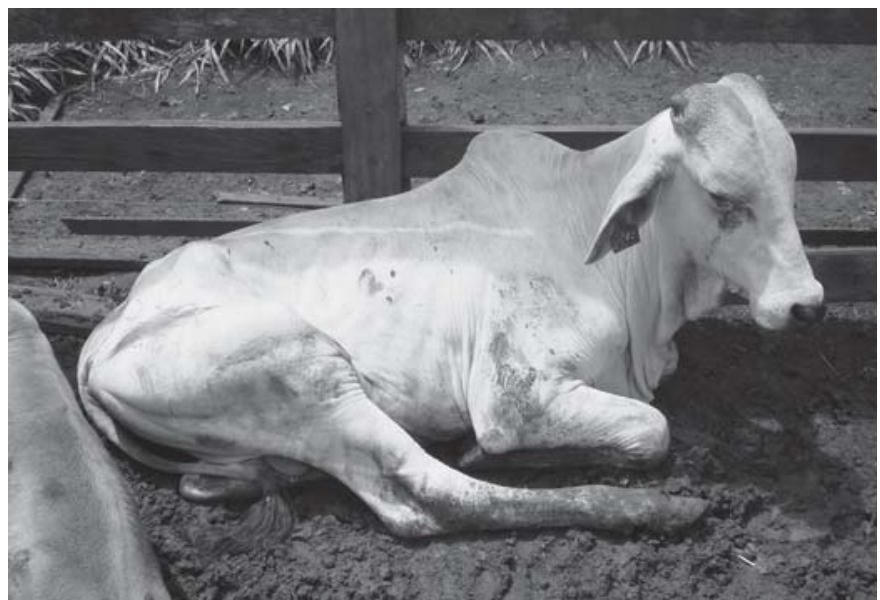

Fig.1. Apatia acentuada na polioencefalomalacia induzida por amprólio no Bovino 8.

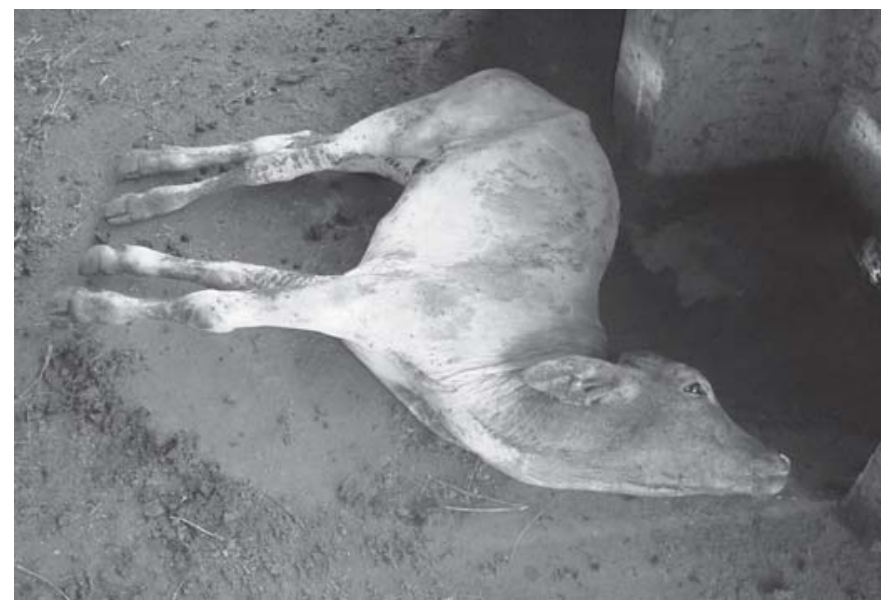

Fig.2. Opistótono na polioencefalomalacia induzida por amprólio no Bovino 9.

vados no Bovino 2. Nos bovinos do Grupo 3, as principais alterações eram restritas ao sistema nervoso central e incluíam tumefação leve do encéfalo e achatamento, amolecimento e amarelamento das circunvoluções cerebrais (Fig.3). Adicionalmente, havia hemorragia focal no gânglio trigêmeo do Bovino 9 e hemorragias multifocais na serosa do omaso e mucosa da bexiga do Bovino 7. Não foram observadas alterações macroscópicas nos Bovinos 5 e 6.

As lesões microscópicas dos bovinos do Grupo 1 incluíam hemorragia focal na medular e cortical dos rins e multifocal leve a moderada no miocárdio e congestão e edema pulmonar difuso moderado. No rúmen, retículo e omaso, áreas acentuadas de hemorragia associadas a infiltrados de neutrófilos foram detectadas na submucosa, muscular e serosa. O Bovino 4 apresentou no retículo áreas focalmente extensas de necrose de coagulação na mucosa associadas a infiltrado de neutrófilos e hemorragia acentuada. No telencéfalo parietal, havia migração de neutrófilos dos vasos da leptomeninge para o espaço perivascular. Não foram observadas alterações histológicas no Bovino 5.

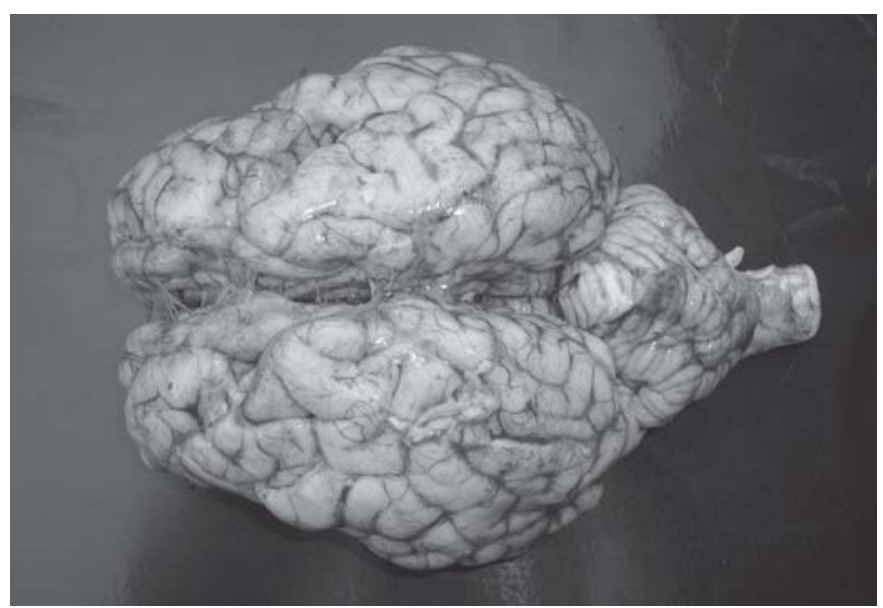

Fig.3. Encéfalo do Bovino 8 com polioencefalomalacia induzida por amprólio. O encéfalo está tumefeito e há achatamento e amarelamento das circunvolunções. 


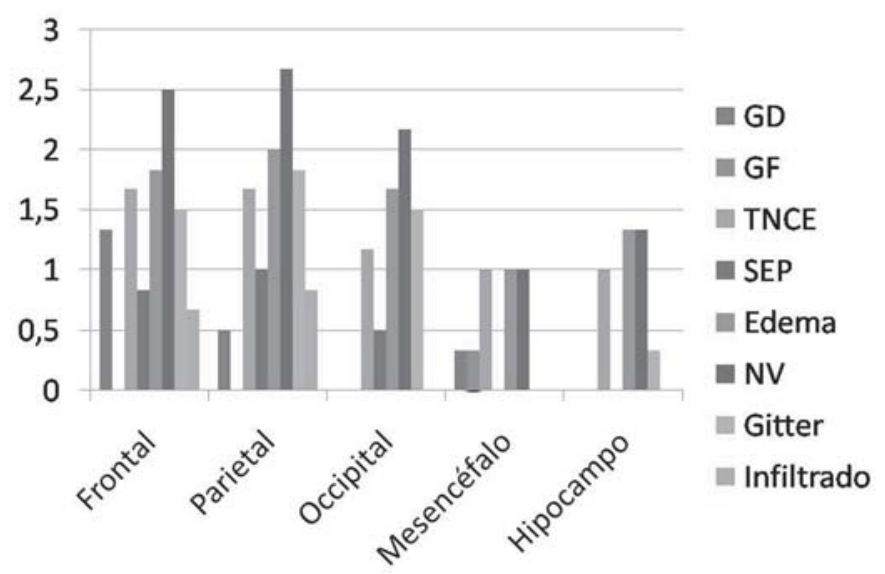

Fig.4. Tipo, distribuição e intensidade das lesões induzidas experimentalmente por amprólio no encéfalo de quatro bovinos. Os números que aparecem na ordenada referemse à intensidade da lesão: 0 = ausência de lesão, 1 = lesão leve, 2 = moderada, 3 = acentuada. Na abscissa estão os locais do encéfalo (telencéfalos frontal, parietal e occipital, mesencéfalo, hipocampo) onde ocorriam as lesões.

No Bovino 6 e nos animais do Grupo 3, as alterações microscópicas estavam restritas ao encéfalo e foram mais acentuadas nos telencéfalos frontal, parietal e occipital. Lesões de intensidade moderada também foram detectadas no

Fig.5. Telencéfalo parietal do Bovino 9 na polioencefalomalacia induzida por amprólio. Há necrose neuronal que têm o citoplasma acidofílico e núcleo picnótico (neurônios vermelhos) e edema perineuronal e do neurópilo (espongiose). HE, obj.40x.

Fig.6. Telencéfalo parietal do Bovino 9 na polioencefalomalacia induzida por amprólio. Há infiltração moderada de macrófagos espumosos (células gitter) na leptomeninge e no neurópilo, neurônios necróticos, tumefação de núcleos de células endoteliais e edema. HE, obj.20x.

Fig.7. Mesencéfalo do Bovino 9. Área focal adjacente ao núcleo do nervo trigêmeo com necrose neuronal (neurônios vermelhos), astrogliose, edema de neurópilo e tumefação de núcleos de células endoteliais. HE, obj.20x.

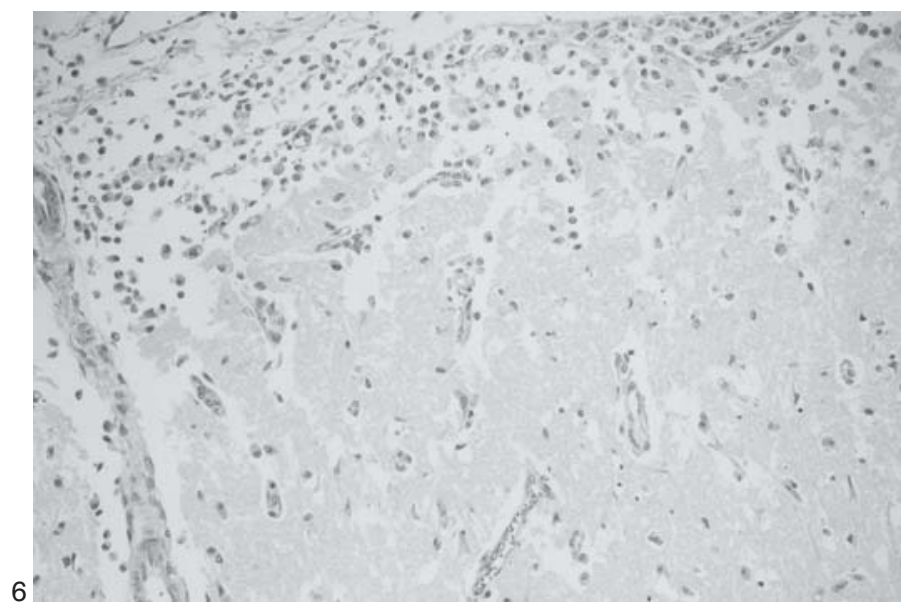

hipocampo e mesencéfalo. A Figura 4 mostra a distribuição e intensidade dessas lesões. Não foram observadas alterações histológicas nos núcleos basais, tálamo, cerebelo, bulbo, ponte e no complexo GRH desses bovinos. As principais alterações microscópicas no córtex dos hemisférios telencefálicos direito e esquerdo foram consistentes em todos os casos e incluíam necrose neuronal segmentar e laminar, edema (espongiose) perineuronal, perivascular e no neurópilo, tumefação de núcleos de células endoteliais dos vasos e ocasional hipertrofia de astrócitos (Fig.5). Em todos os casos, numerosos macrófagos tumefeitos com citoplasma espumoso (células gitter) eram observados principalmente na leptomeninge e no espaço perivascular e neurópilo do córtex (Fig.6). Os neurônios necróticos eram caracterizados por encarquilhamento e hipereosinofilia citoplasmáticas, picnose nuclear sem evidenciação nucleolar e desaparecimento da substância de Nissl. Ocasionalmente, os núcleos dos neurônios necróticos apresentavam aspecto vítreo. A necrose neuronal ocorreu tanto nos topos quanto nos sulcos das circunvoluções cerebrais. O edema na substância cinzenta era acentuado e, em alguns casos, deixava o neurópilo com aspecto esponjoso; ocorria comumente separando a substância cinzenta da branca ou as camadas de neurônios da substância cinzenta. Em dois bovinos, havia manguitos perivasculares focais ou multifocais leves de linfócitos e macrófagos. Gliose focal ou difusa e congestão foram observa-

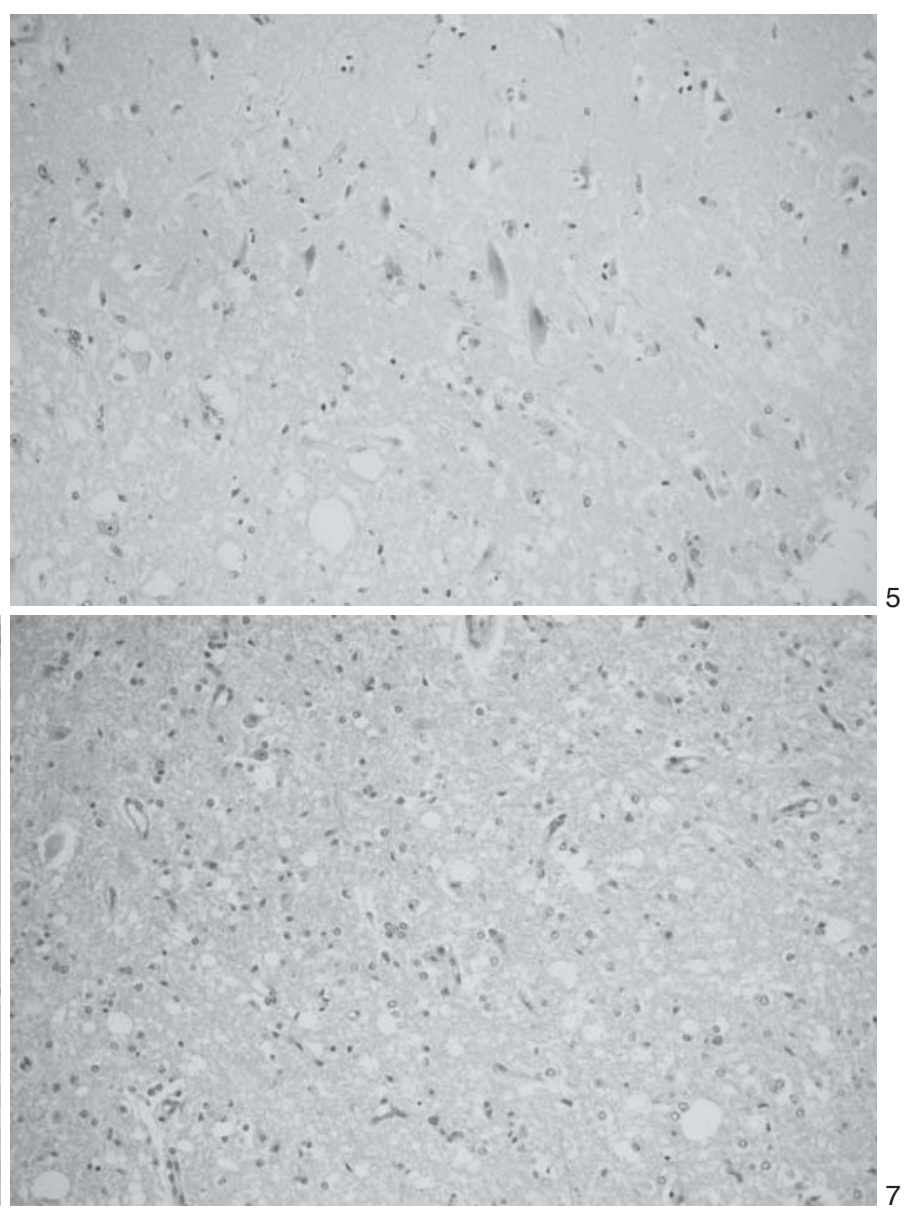


das com pouca frequência. Em nenhum caso foi observada neuronofagia, hemorragia e ou lesão residual (cicatriz). Lesões no mesencéfalo ocorreram em dois bovinos, eram leves ou moderadas e restritas ao teto do mesencéfalo e a áreas adjacentes ao núcleo do nervo trigêmeo. Nessa última região, as lesões eram simétricas e bilaterais e caracterizavam-se por áreas com necrose neuronal focal, edema de neurópilo, tumefação de núcleos de células endoteliais e astrogliose (Fig.7). No hipocampo, as alterações eram muito similares às do mesencéfalo e incluíam, adicionalmente, esferóides axonais focais. A distribuição da necrose neuronal e do edema nas camadas de neurônios da substância cinzenta dos lobos frontal, parietal e occipital do telencéfalo, não diferiu entre esses bovinos, afetando uniformemente todas as camadas.

\section{DISCUSSÃO}

No presente estudo, dois quadros morfológicos foram observados em bovinos intoxicados por amprólio. O primeiro foi observado nos bovinos que receberam $1.000 \mathrm{mg} / \mathrm{kg} \mathrm{e} \mathrm{em}$ um que recebeu $500 \mathrm{mg} / \mathrm{kg}$ e caracterizou-se por hemorragias difusas, principalmente no trato digestivo. $O$ segundo quadro detectado nos bovinos que ingeriram $350 \mathrm{mg} / \mathrm{kg}$ de amprólio e em um que ingeriu $500 \mathrm{mg} / \mathrm{kg}$, consistiu de quadro neurológico caracterizado por polioencefalomalacia e hemorragias de menor intensidade em alguns órgãos. No Grupo 1, os animais morreram precocemente sem desenvolverem PEM, enquanto que no Grupo 3 e no Bovino 6, PEM foi reproduzida após 22-28 dias de administração de amprólio e curso clinico de 4-7 dias. Bezerros que receberam dieta com $1 \%$ de amprólio desenvolveram PEM com 25-39 dias de experimentação (Markson et al. 1974). Em outro estudo, bezerros que ingeriram diariamente $321-418 \mathrm{mg} /$ $\mathrm{kg}$ de amprólio (produto com $20 \%$ de amprólio), apresentaram sinais clínicos e lesões de PEM 24-49 dias após o início do experimento (Kasahara et al. 1989). Já ovinos que receberam $500 \mathrm{mg} / \mathrm{kg}$ e $1.000 \mathrm{mg} / \mathrm{kg}$ reproduziram PEM após 28-59 e 13-39 dias de administração de amprólio, respectivamente (Sant'Ana et al. 2009c).

Os sinais clínicos neurológicos apresentados pelos bovinos deste estudo estão associados às lesões corticais e ao edema que causou compressão do tronco encefálico e de outras estruturas ventrais do encéfalo e foram semelhantes aos relatados em outras descrições de PEM associada às intoxicações por amprólio (Markson et al. 1974, Kasahara et al. 1989, Sant'Ana et al. 2009c) e por enxofre (Sager et al. 1990, Jeffrey et al. 1994, Niles et al. 2000) em ruminantes. Os sinais clínicos agudos a subagudos observados nos bovinos do Grupo 1 não têm sido descritos na intoxicação experimental por amprólio em bovinos (Markson et al. 1972, Kasahara et al. 1989) ou em ovinos (Loew \& Dunlop 1972, Morgan 1974, Morgan et al. 1975, Sant'Ana et al. 2009c). A dose de amprólio utilizada nesse grupo $(1.000 \mathrm{mg} / \mathrm{kg})$ foi alta o bastante para matar os bovinos sem, entretanto, desenvolver sinais e lesões compatíveis com PEM.

As alterações macroscópicas do encéfalo observadas neste estudo são semelhantes às descritas em outros tra- balhos de PEM em bovinos (Jeffrey et al. 1994, Nakazato et al. 2000, Sant'Ana et al. 2009a). Em alguns casos de PEM nessa espécie, não são observadas lesões macroscópicas (Nakazato et al. 2000, Schild et al. 2005). Áreas de hemorragia no encéfalo são relatadas em PEM associada às intoxicações por amprólio em ovinos (Morgan 1974, Morgan et al. 1975, Sant'Ana et al. 2009c) ou, ocasionalmente, na toxicose por enxofre em bovinos (Hamlen et al. 1993). Na PEM relacionada à intoxicação por amprólio em ovinos, hemorragia parece ser uma alteração importante, uma vez que a mesma não ocorre com frequência na doença associada a outras causas (Sant'Ana et al. 2009c). No bovino, essa lesão encefálica não tem sido observada nessa intoxicação (Markson et al. 1972, Kasahara et al. 1989). A patogenia para a formação das hemorragias na PEM relacionada ao amprólio não é bem esclarecida. Alguns pesquisadores acreditam que o amprólio pode desencadear trombocitopenia secundária a degeneração de megacariócitos na medula óssea em ovinos (Morgan et al. 1975). No presente trabalho, a avaliação histológica da medula óssea e o plaquetograma não foram realizados para esclarecer essa hipótese.

Embora as lesões histológicas tenham sido mais intensas nos lobos frontal, parietal e occipital, alterações moderadas também foram detectadas no mesencéfalo e hipocampo. Esse tipo de lesão cortical e de estruturas mais ventrais do encéfalo é considerada uma forma grave da doença e tem sido observada na PEM em bovinos do Brasil (Sant'Ana et al. 2009a) e de outros países associada ao consumo excessivo de enxofre (Hamlen et al. 1993, Hill \& Ebbett 1997, Gould 2000). Entretanto, nesses casos causados por enxofre, há lesões vasculares como degeneração de veias e vênulas (Loneragan et al. 1998) e necrose fibrinóide de arteríolas no tálamo (Hamlen et al. 1993) e mesencéfalo (Hamlen et al. 1993, Hill \& Ebbett 1997), o que não foi observado na intoxicação por amprólio do presente estudo e em outro estudo similar realizado em ovinos (Sant'Ana et al. 2009c). No presente estudo, o padrão de necrose neuronal era laminar e segmentar, semelhante ao observado em outros casos naturais de PEM (Nakazato et al. 2000, Sant'Ana et al. 2009a). Na intoxicação por amprólio em ovinos, o limite entre a substância cinzenta afetada por neurônios necróticos e a íntegra é abrupto (Sant'Ana et al. 2009c), o que não tem sido encontrado na PEM relacionada a outras causas (Barros et al. 2006).

A necrose neuronal e o edema ocorreram homogeneamente entre as lâminas de neurônios do córtex telencefálico, semelhantemente ao observado em ovinos intoxicados experimentalmente por amprólio (Morgan 1974, Sant'Ana et al. 2009c) e naturalmente por enxofre (Bulgin et al. 1996, Low et al. 1996). Em casos espontâneos em bovinos, as camadas granular externa e interna do córtex occipital, parietal e frontal foram mais afetadas por necrose de neurônios e edema (Sant'Ana et al. 2009a). Na PEM associada à intoxicação por enxofre em bovinos, a necrose predomina nas camadas corticais superiores e médias de neurônios piramidais (Hamlen et al. 1993, Jeffrey et al. 
1994) e a espongiose ocorre especialmente nas camadas profundas (Gould 2000).

O conhecimento das doses de amprólio necessárias para o estabelecimento de PEM em bovinos, a determinação dos sinais clínicos, da evolução da doença, do tipo e da distribuição das lesões no sistema nervoso central, permite estabelecer um modelo experimental eficiente em bovinos para o estudo da PEM em ruminantes.

\section{REFERÊNCIAS}

Barros C.S.L., Driemeier D., Dutra I.S. \& Lemos R.A.A. 2006. Doenças do Sistema Nervoso de Bovinos no Brasil. Agnes, São Paulo, p.166171.

Bulgin M.S., Lincoln S.D., Mather G. 1996. Elemental sulfur toxicosis in a flock of sheep. J. Am. Vet. Med. Assoc. 208:1063-1065.

Gould D.H. 2000. Update on sulfur-related polioencephalomalacia. Vet. Clin. North Am., Food Anim. Pract. 16:481-496.

Hamlen H., Clark E. \& Janzen E. 1993. Polioencephalomalacia in cattle consuming water with elevated sodium sulfate levels: A herd investigation. Can. Vet. J. 34:153-158.

Hill F.I. \& Ebbett P.C. 1997. Polioencephalomalacia in cattle in New Zealand fed chou moellier (Brassica oleracea). N. Z. Vet. J. 45:37-39.

Innes J.R.M. \& Saunders L.Z. 1962. Encephalomalacia and myelomalacia, p.607-609. In: Ibid. (Eds), Comparative Neuropathology. Academic Press, New York. 839p.

Jeffrey M., Duff J.P., Higgins R.J., Simpson V.R., Jackman R., Jones T.O., Mechie S.C. \& Liversey C.T. 1994. Polioencephalomalacia associated with the ingestion of ammonium sulphate by sheep and cattle. Vet. Rec. 134:343-348.

Jensen R., Griner L.A. \& Adams O.R. 1956. Polioencephalomalacia of cattle and sheep. J. Am. Vet. Med. Assoc. 129:311-321.

Kasahara T., Ichijo S., Osame S. \& Sarashina T. 1989. Clinical and biochemical findings in bovine cerebrocortical necrosis produced by oral administration of amprolium. Jpn. J. Vet. Sci. 51:79-85.

Loew F.M. \& Dunlop R.H. 1972. Induction of thiamine inadequacy and polioencephalomalacia in adult sheep with amprolium. Am. J. Vet. Res. 33:2195-2205.

Loneragan G.H., Gould D.H., Callan R.J., Sigurdson C.J. \& Hamar D.W. 1998. Association of excess sulfur intake and an increase in hydrogen sulfide concentrations in the ruminal gas cap of recently weaned beef calves with polioencephalomalacia. J. Am. Vet. Med. Assoc. 213:1599-1604.

Low J.C., Scott P.R., Howie F., Lewis M., FitzSimons J. \& Spence J.A. 1996. Sulphur-induced polioencephalomalacia in lambs. Vet. Rec. 138:327-329.

Markson L.M., Lewis G., Terlecki S., Edwin E.E. \& Ford J.E. 1972. The aetiology of cerebrocortical necrosis: The effects of administering antimetabolites of thiamine to preruminant calves. Brit. Vet. J. 128:488499.

Markson L.M., Edwin E.E., Lewis G. \& Richardson C. 1974. The production of cerebrocortical necrosis in ruminant calves by the intraruminal administration of amprolium. Brit. Vet. J. 130:9-16.

Morgan K.T. 1974. Amprolium poisoning of preruminant lambs: An ultrastructural study of the cerebral malacia and the nature of the inflammatory response. J. Pathol. 112:229-236.

Morgan K.T., Coop R.L. \& Doxey D.L. 1975. Amprolium poisoning of preruminant lambs. An investigation of the encephalopathy and the haemorrhagic and diarrhoeic syndromes. J. Pathol. 116:73-81.

Nakazato L., Lemos R.A.A. \& Riet-Correa F. 2000. Polioencefalomalacia em bovinos nos estados de Mato Grosso do Sul e São Paulo. Pesq. Vet. Bras. 20:119-125.

Niles G.A., Morgan S.E. \& Edwards W.C. 2000. Sulfur-induced polioencephalomalacia in stocker calves. Vet. Human Toxicol. 42:290-291.

Sager R.L., Hamar D.W. \& Gould D.H. 1990. Clinical and biochemical alterations in calves with nutritionally induced polioencephalomalacia. Am. J. Vet. Res. 51:1969-1974.

Sant'Ana F.J.F., Rissi D.R., Lucena R.B., Lemos R.A.A., Nogueira A.P.A. \& Barros C.S.L. 2009a. Polioencefalomalacia em bovinos: epidemiologia, sinais clínicos e distribuição das lesões no encéfalo. Pesq. Vet. Bras. 29:487-497.

Sant'Ana F.J.F., Lemos R.A.A., Nogueira A.P.A., Togni M., Tessele B. \& Barros C.S.L. 2009b. Polioencefalomalacia em ruminantes. Pesq. Vet. Bras. 29:681-694.

Sant'Ana F.J.F., Nogueira A.P.A., Souza R.I.C., Cardinal S.G., Lemos R.A.A. \& Barros C.S.L. 2009c. Polioencefalomalacia experimental induzida por amprólio em ovinos. Pesq. Vet. Bras. 29:747-752.

Schild A.L., Ferreira J.L.M. \& Soares M.P. 2005. Polioencefalomalácia em bovinos. Bolm 25, Laboratório Regional de Diagnóstico, Pelotas, RS, p.32-34. 